\title{
Energy harvesting floor from commercial cellulosic materials for self-powered wireless transmission sensor system
}

Long Gu, ${ }^{1, \digamma}$ Lazarus German, $, 1,2,{ }^{1}$ Tong Li, ${ }^{1}$ Jun $\mathrm{Li},{ }^{1}$ Yan Shao, ${ }^{1}$ Yin Long, ${ }^{1}$ Jingyu Wang, ${ }^{1}$ Xudong Wang ${ }^{1, *}$

1. Department of Materials Science and Engineering, University of WisconsinMadison, Madison, WI, 53706, USA

2. EWPanel, LLC, Madison, WI, 53715, USA

$\dagger$ Authors with equal contribution.

* Corresponding author Email: xudong.wang@wisc.edu 


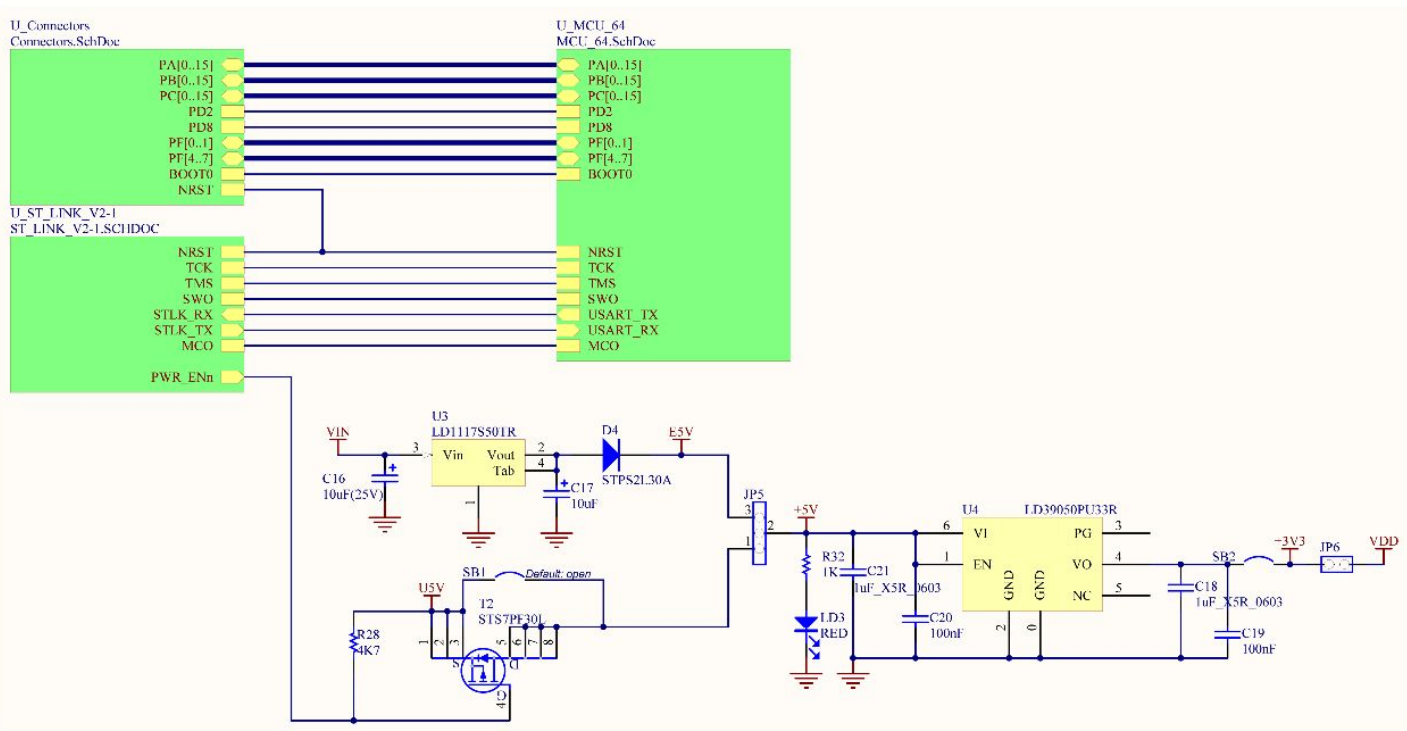

Figure S1. Circuit diagram of the wireless transmission sensing system.

(a)

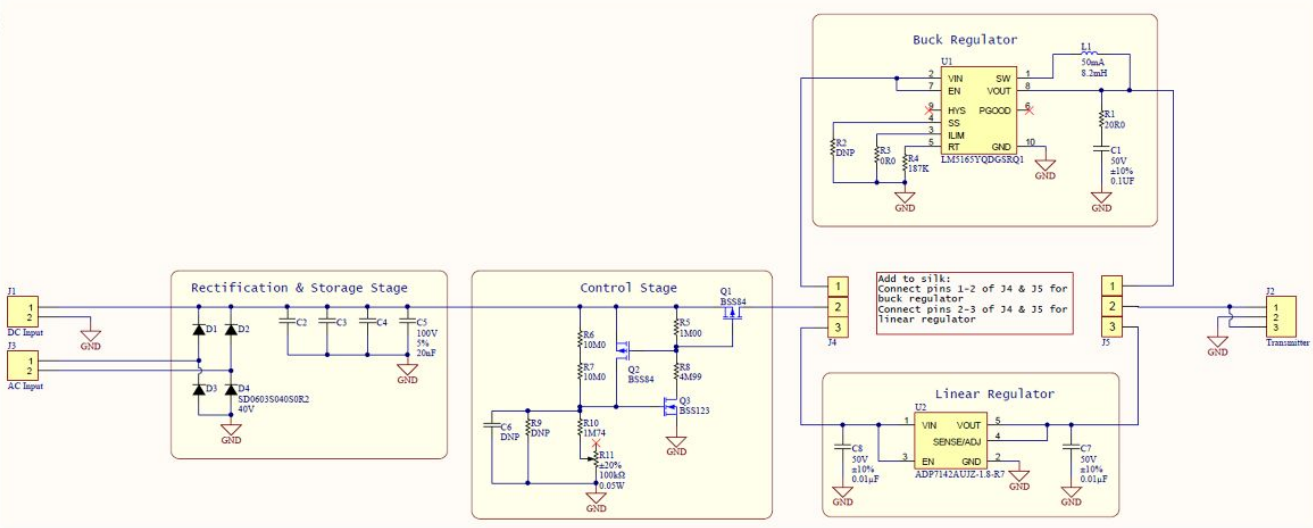

(b)

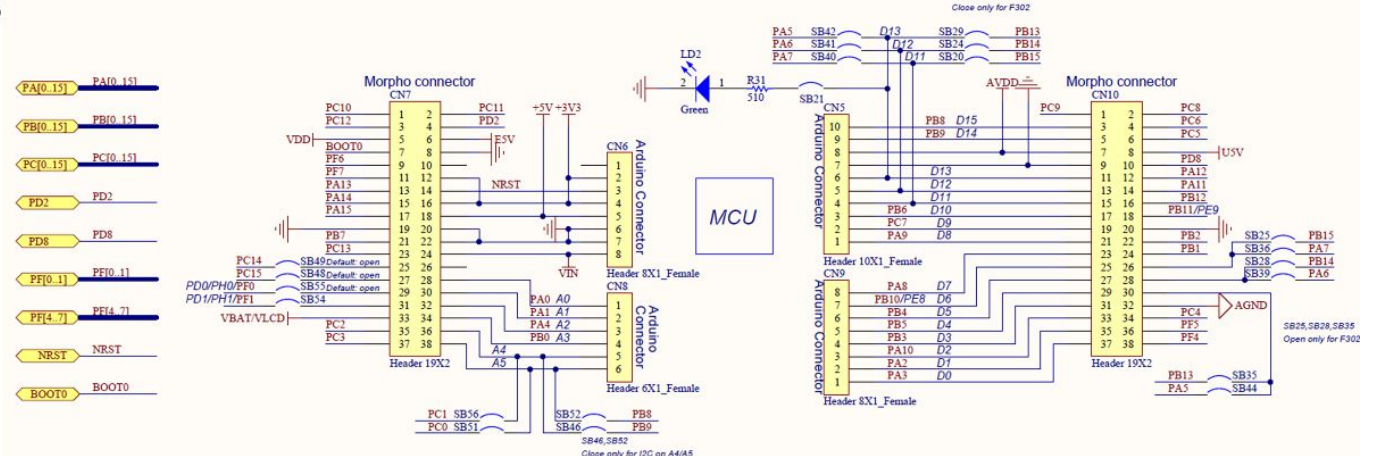

Figure S2. Circuit diagrams of the transmitter (a) and (b) receiver. 


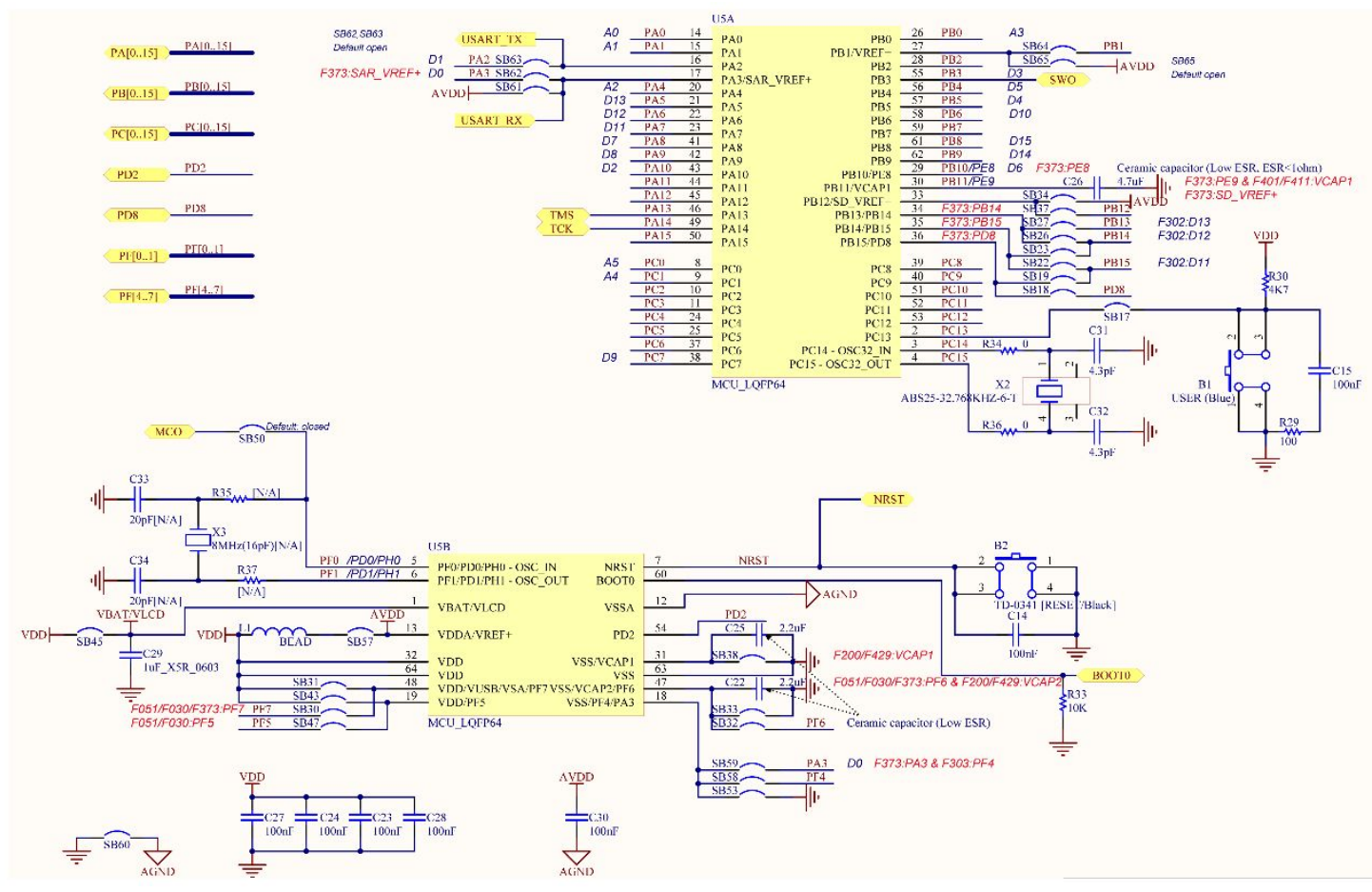

Figure S3. Circuit diagram of the micro controller unit (MCU) in receiver. 